\title{
Effect of electric fields in low-dimensional materials: Nanofrictional response as a case study
}

\author{
Florian Belviso ${ }^{\circ},{ }^{*}$ Antonio Cammarata ${ }^{\dagger},{ }^{\dagger}$ and Jamil Missaoui 10 \\ Department of Control Engineering, Faculty of Electrical Engineering, \\ Czech Technical University in Prague, Technicka 2, 16627 Prague 6, Czech Republic
}

\author{
Tomas Polcar (1) \\ Engineering Materials \& nCATS, FEE, University of Southampton, SO17 1BJ Southampton, United Kingdom
}

(Received 28 August 2020; revised 11 October 2020; accepted 12 October 2020; published 29 October 2020)

\begin{abstract}
A proper control of nanoscale friction is mandatory for the fabrication and operation of optimal nanoengineered devices. In this respect, the use of electric fields looks to be promising, since they are able to alter the frictional response without imprinting permanent deformations into the structure. To this aim, we perform $a b$ initio simulations to study the microscopic mechanisms governing friction in low-dimensional materials in the presence of electrostatic fields. We consider $\mathrm{MX}_{2}$ transition metal dichalcogenides as a case study. By applying an electric field along an axis orthogonal to the atom layers, we induce a transfer of charge along the same axis; this transfer modifies the interatomic forces, leading, in general, to easier relative layer motion. The reported outcomes constitute a starting point to study the effect of the field direction on the intrinsic friction in future investigations. Finally, the present results can be used to predict the preferential electronic redistribution in nanostructured devices where metal-to-insulator transitions may occur in working conditions.
\end{abstract}

DOI: 10.1103/PhysRevB.102.155433

\section{INTRODUCTION}

Low-dimensional materials have found vast use in nanoengineered devices thanks to their properties with wide applicability such as in photovoltaics, lithium ion batteries, hydrogen evolution catalysis, transistors, photodetectors, DNA detection, memory devices, and tribology [1-5]. Fabrication of such devices requires micromanipulation of free standing atomic layers, and a thorough knowledge and control of the frictional properties at the nanoscale is then mandatory [6,7]. Moreover, their frictional properties are of particular interest in micro- and nanoelectromechanical devices [8,9] (MEMS, NEMS) or in energy harversters, where nanoscale layers are in relative motion $[9,10]$. Previous studies already pointed out that load can be used as an external knob to control the nanoscale friction [11-13], although it may induce unwanted permanent changes to the atomic geometry [14-17]. On the other hand, electrical fields induce charge movements which may alter the frictional properties temporarily, while allowing them to revert to the original structure once the external field is suppressed [18-20]. Some theoretical studies have already dealt with the effect of electric fields on the coupling between the electronic and dynamic (i.e., phonon) features in low-dimensional materials [21,22]; interestingly, such coupling has been found to be effective in altering the lateral frictional force in $\mathrm{MoS}_{2}$ and graphene bilayer systems $[23,24]$. Recent experimental studies showed how to manipulate free-standing atomic layers by in-plane

\footnotetext{
*belviflo@fel.cvut.cz

†cammaant@fel.cvut.cz
}

potential gradients with an atomic force microscopy tip [6,25], while the friction between the tip and the layer decreases with the field [20].

Motivated by this findings, in the present work we study the microscopic mechanisms governing the intrinsic friction at the nanoscale in the presence of electrostatic fields. As a case study, we consider van der Waals transition metal dichalcogenides (TMDs), which are of great interest in diverse fields where nanoengineering is the fundamental used technique [2]. We use quantum mechanical simulations to uncover the electronic response to an external field; we then show how this determines the atomic participation to the relative motion of few TMD layers and hence the intrinsic friction. The outcomes constitute a theoretical tool for future studies on the effect of the field direction on the layer motions. Among other applications, we finally discuss how the present results can be exploited in machine learning engines aimed to design nanotribological materials with targeted frictional response.

\section{METHODS}

\section{A. Computational details}

The bulk structure of $\mathrm{MX}_{2}$ transition metal dichalcogenides consists of hexagonally-ordered planes of $\mathrm{M}$ cations, inserted between two planes of $\mathrm{X}$ anions arranged in a similar fashion. This layered motif produces X-M-X "sandwiches" held together by van der Waals forces and yielding a lamellar structure. Such forces are weak enough to allow easy relative sliding of neighboring layers, manifesting as macroscopic lubricating effect. Among the possible polymorphs [1], we consider the $2 \mathrm{H}$ phase with space group symmetry $P 6_{3} / \mathrm{mmc}$, 
(a)
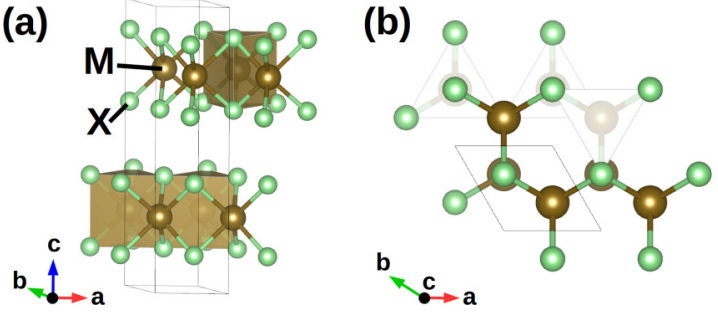

FIG. 1. Model geometry of the hexagonal $P 6_{3} / m m c 2 \mathrm{H}$ polymorph of prototypical TMD compounds; the unit cell is indicated by a fine-line box. The $\boldsymbol{a}, \boldsymbol{b}, \boldsymbol{c}$ vectors forming the reference frame at the bottom left of each subfigure represent the crystallographic axes in our settings and the orientation of the unit cell with respect to the reader's view. Adjacent $\mathrm{MX}_{2}$ layers are bound together by weak van der Waals forces which allow relative gliding.

in which the anions are aligned with the cations along a direction orthogonal to the layer surface (Fig. 1). We select $\mathrm{M}=\mathrm{Mo}, \mathrm{W}$ and $\mathrm{X}=\mathrm{S}, \mathrm{Se}, \mathrm{Te}$; in our settings, the layers are able to slide in parallel with the $a, b$ plane.

We perform density functional theory calculations by means of the ABINIT software [26-32]. The plane-wave energy cutoff is set to a minimum of $18.4 \mathrm{Ha}$ and the Brillouin zone is sampled with a minimum of $7 \times 7 \times 5 k$-mesh divisions; the convergence of the electronic distribution is considered to be achieved when the difference of the total energy calculated between two subsequent self-consistent field cycles occurs twice in a row to be less than $10^{-12} \mathrm{Ha}$. Structural relaxations are initiated from diffraction data [33-38]; the lattice parameters and atomic positions are considered to be optimized when the maximum component of the total force acting on each atom is less than $10^{-7} \mathrm{Ha} / \mathrm{Bohr}$.

After a preliminary benchmark, [39] we select the PBE functional [40] together with the vdw-DFT-D3(BJ) correction [41] to account for the van der Waals interactions. Following the formulation of Nunes and Gonze $[42,43]$, we apply an external electrostatic field $\boldsymbol{E}$ along a direction parallel to the $c$ lattice vector (perpendicular to the sliding plane), the magnitude of which varies in the range $[0,2.5] \times 10^{-3}$ a.u. Apart from the largest values of 2.0 and $2.5 \times 10^{-3}$ a.u. which we consider as extreme cases, the remaining values are representative of the electric fields typically used in MEMS/NEMS devices [44-48]. We observe the presence of structural instabilities beyond certain field values depending on the atomic types forming the structure; for this reason, the maximum field value is chosen differently for each system in order to minimize the number of unstable displacements. We finally calculate eigendisplacements and eigenfrequencies of the dynamical matrix of the structures by using the PHONOPY software [49].

\section{B. Sliding and breathing modes}

Microscopic friction is defined as the friction appearing due to the relative motion of adjacent atom layers in the presence of structural irregularities (e.g., dislocations, layer truncations, wrinkles). If such irregularities are absent, friction is still occurring due to the interatomic forces arising from the electronic (type of atom) and structural arrangement (geometry); in this case, we call it intrinsic friction as it is an intrinsic characteristic of the system [50]. In the present work, we focus on the latter by considering pristine compounds lacking in irregularities or defects. We already observed that relative atomic motions can be geometrically described by linear combination of phonon eigendisplacements [51,52]; those with the largest coefficients in the combination are named sliding modes and keep active the layer sliding as long as they own enough energy $[53,54]$. The sliding modes can be classically seen as restoring forces which prevent the layer to displace from the equilibrium position, such forces being proportional to the square of the mode frequency. Therefore, at a constant system energy, the lower the frequency of the sliding modes, the larger the amplitude of the corresponding atomic displacements, this corresponding to facilitated sliding. If the frequency of a sliding mode becomes negative [55] due to external stimuli, the geometry becomes unstable against the distortion represented by the eigendisplacement of such a mode, and the atomic configuration does not realize a minimum of the energy anymore. This means that a very small perturbation of the geometry is enough to activate the sliding motion along the direction of the mode eigenvector; as a consequence, a new minimum of the energy is realized. The latter corresponds to a new geometric configuration with new sliding mode frequencies which, in principle, can be larger than the initial ones. To obtain easy sliding over long distances, it is then necessary to lower the frequencies of the sliding modes at each local minimum of the energy landscape; indeed, it has been found that layered compounds display several energy minima with different curvatures [56-60]. Nevertheless, the present discussion is general and does not depend on the specific atomic topology. For this reason, we here focus on the structural configurations corresponding to one of the possible energy local minima of the considered compounds; the same analysis can then be repeated on the geometries corresponding to any other local energy minimum. If an approximate evaluation of the frequencies is required, it is possible to reduce the computational load by exploiting the normal-modes transition approximation [52]: Starting from the knowledge of the phonon modes of one of the stable structures, the approximation allows us to estimate the eigenfrequencies of neighboring energy minima, thus avoiding the time-consuming diagonalization of the corresponding dynamical matrices.

In tribological conditions, external intervention produces displacements of a layer with respect to the adjacent ones; such displacements generate an excessive overlap of the electronic densities of the facing anions, and a consequent repulsive force arising from both Coulombic interactions and Pauli's exclusion principle. Since the external forces drag the layers along directions parallel to the $a, b$ plane, the effect of the repulsive forces is to push the layers away from each other along the $\boldsymbol{c}$ direction orthogonal to the layer plane. In the extreme case in which the orthogonal movement is not allowed, the repulsive force would act only laterally pushing the layer back, then increasing the sliding energy barrier. It is then apparent that if the forces binding the layers together are weak, the layer separation is facilitated and hence the sliding. The latter forces mainly arise from the van der Waals interactions and are represented by the breathing phonon modes. The breathing modes are associated to a restoring force which 
regulates the interlayer distance: A small force corresponds to a large allowed variation (compression/dilation) of the layer separation and facilitates the lateral shift. Both sliding and breathing motions then occur at the same time whenever two layers are displaced, no matter if the displacement is done slowly and in a reversible way (case of static friction) or rapidly (case of dynamic friction); in terms of the phonon description, this corresponds to the phonon coupling between both kind of modes, leading to dissipative processes which are active until the relative layer displacement occurs $[53,54]$. By tuning the phonon frequency we can then control the intrinsic frictional response; to this aim, in what follows, we will focus on both the sliding and the breathing modes. A schematic representation of the displacements associated to such modes is reported, for example, in the freely available Supplemental Material of Ref. [50] and in Ref. [19].

We want to avoid the fragmentation of information into several parameters; instead, we use global quantities in order to obtain a unified description of the physics of the system. To this aim, we consider the mean frequency $\omega$ which is calculated as

$$
\omega=\frac{1}{N} \sum_{j}^{N} \omega_{j},
$$

where $N$ is the number of the considered sliding and breathing modes and $\omega_{j}$ is the frequency of the $j$ th mode. It is worth noting here that $\omega$ can assume positive values despite the fact that some of the $\omega_{j}$ can be negative, that is, also in the presence of unstable modes. In this respect, a positive value of $\omega$ indicates that the amount of instabilities, that is the number of unstable modes and the magnitude of the relative frequencies, is null of negligible; in the latter case, the structure can be considered "globally" stable. The phonons relevant to our discussion are mainly located at the $\Gamma$ and $A$ points of the irreducible Brillouin zone; we will then focus on this portion of the reciprocal space in our phonon analysis.

\section{RESULTS AND DISCUSSION}

We start our investigation from the effect of the electric field on the geometry. At non-null fields, we observe the largest volume variation in the $\mathrm{MoSe}_{2}, \mathrm{MoTe}_{2}$, and $\mathrm{WSe}_{2}$ systems, mainly due to the change in the length of the $c$ lattice vector (Fig. 2); this is expected, since such a crystallographic axis is parallel to the direction of the applied field $\boldsymbol{E}$. As we discuss later on, some structure becomes unstable for field values beyond a certain threshold which depends on the material; for those values, the lattice parameters correspond to a transient configuration which belongs to a geometric path connecting two stable states. Such transient configuration can be detected by time-resolved crystallography techniques, capable to monitor structural changes occurring in very short time scales [61-63]. Concerning the phonon structure, we observe that an increase of the field produces a general hardening of the $\Gamma-A$ modes together with a softening of some sliding branches, which may become unstable beyond some critical $E(E=|\boldsymbol{E}|)$ value specific of the system. This is apparent when we consider the average frequency $\omega$ as a function of $E$ (Fig. 3): The $\mathrm{MoS}_{2}, \mathrm{WS}_{2}$, and $\mathrm{WTe}_{2}$ systems appear to

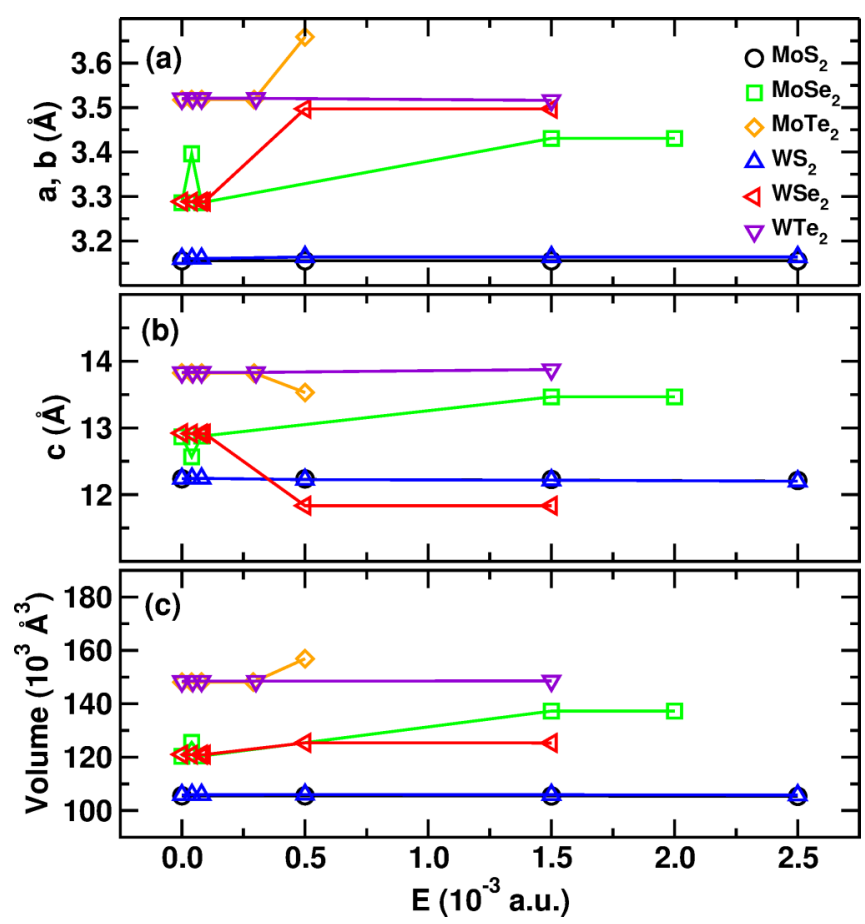

FIG. 2. Effect of the electric field on the lattice parameters of the model systems: (a) lattice constants $a$ and $b$, (b) lattice constant $c$, (c) cell volume. Values relative to $\mathrm{MoS}_{2}$ and $\mathrm{WS}_{2}$ systems are close. Lines are a guide for the eye.

be the most stable against large field perturbations while, in the remaining compounds, some of the sliding modes become unstable already at $E=0.5 \times 10^{-3}$ a.u. (the dependency of each sliding and breathing mode frequency as a function of the field is reported in the Supplemental Material [39]). This suggests that the sliding can already be facilitated in $\mathrm{MoSe}_{2}$, $\mathrm{MoTe}_{2}$, and $\mathrm{WSe}_{2}$ pristine TMDs by means of the application of small electric fields; however, the critical electric fields to obtain easy sliding are the lowest required to realize negative average frequencies and depend on the chemical composition. To estimate the effect of the field on the nanoscale friction, we recall that we are here describing friction as a harmonic restoring force

$$
f=-k x=m \omega^{2} \ddot{x},
$$

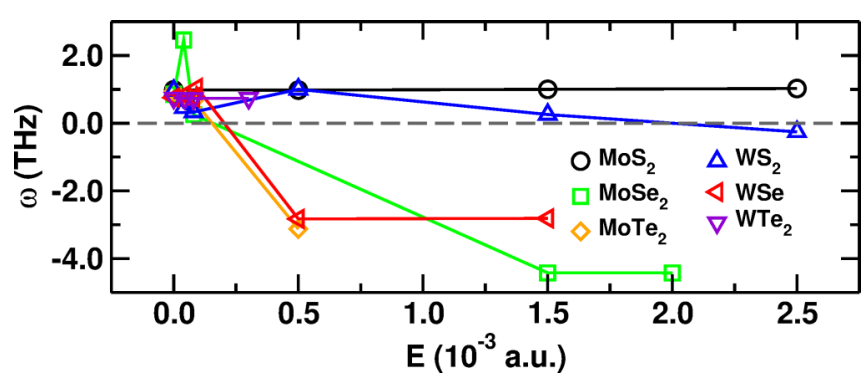

FIG. 3. Average frequency as a function of the applied electric field. Instabilities arise when the field magnitude is above a critical value depending on the chemical composition. Lines are a guide for the eye. 
where $x$ is the relative layer displacement, $m$ is the mass of the system, and $k(\omega)$ is an effective force constant (frequency) resulting from the sliding and breathing modes. From Eq. (2) we can infer that, if the effect of the electric field is to halve the frequency, the resulting frictional force is four times smaller than the initial one. We are aware that this is an approximation because phonon coupling should be taken into account explicitly [52-54,64]. A rigorous derivation of the frictional force in terms of phonon contributions requires the explicit expression of the energy in terms of single phonons and the relative coupling, also including the effect of the temperature; however, this falls out of the scope of the present discussion and we plan to investigate it in a future work. By inspecting Fig. 3, it is apparent that there is no clear relation between the magnitude of the field, the phonon frequency, and the atomic types. This relation is the result of the coupling between the electronic structure and the dynamical response of the system; understanding and controlling such coupling is indeed the goal of the present work. To this aim, we proceed with the analysis of the subtle features of the electronic density. To quantify how the electronic charge redistributes across the structure under the effect of the field we make use of the orbital polarization [65] defined as

$$
\mathcal{P}_{a, b}=\frac{n_{a}-n_{b}}{n_{a}+n_{b}},
$$

where $n_{i}$ is the occupation of a given $i$ orbital; in this way, we measure the excess of population of the $a$ with respect to the $b$ orbital. Since the atomic orbitals provide a partition of the electronic density into regions with specific spatial orientations, by comparing different kind of polarizations we can determine the preferential distribution of the electrons at specific field magnitudes. We consider the $p_{x}, p_{y}$, and $p_{z}$ orbitals centered at the anion sites, and the $t_{2 g}$ and the $e_{g}$ orbitals centered at the cation sites, in order to calculate the following polarizations: $\mathcal{P}_{p_{x}, p_{y}}, \mathcal{P}_{p_{x}, p_{z}}, \mathcal{P}_{p_{y}, p_{z}}, \mathcal{P}_{t_{2 g}, e_{g}}, \mathcal{P}_{d_{x^{2}-y^{2}}, d_{z^{2}}}$. We notice that the presence of the field does not induce any significant change in the relative occupation of the $p_{x}$ with respect to the $p_{y}$ orbital [Fig. 4(a)]; the fluctuation around zero of $\mathcal{P}_{p_{x}, p_{y}}$ indicates that the electrons are equally distributed between the two orbitals. This is an expected behavior thanks to the symmetries present in the $a, b$ plane; for the same reason, both $\mathcal{P}_{p_{x}, p_{z}}$ and $\mathcal{P}_{p_{y}, p_{z}}$ orbital polarizations show the same trend [Figs. 4(b) and 4(c)]. The negative values of $\mathcal{P}_{p_{x}, p_{z}}$ and $\mathcal{P}_{p_{y}, p_{z}}$ indicate an excess of electrons along an axis orthogonal to the layers at the anion site, such excess remaining nearly constant irrespective of the applied field. Concerning the relative occupation of the $t_{2 g}$ and $e_{g}$ orbitals, in the $\mathrm{MoSe}_{2}$, $\mathrm{MoTe}_{2}$, and $\mathrm{WSe}_{2}$ systems we notice that an increase of the field induces a transfer of electrons towards the $e_{g}$ orbitals [Fig. 4(d)] and, correspondingly, an electron flow from the $d_{x^{2}-y^{2}}$ to the $d_{z^{2}}$ orbital [Fig. 4(e)]. This points at a charge transfer along an axis orthogonal to the $a, b$ plane and passing through the cation sites. Differently, in the $\mathrm{MoS}_{2}, \mathrm{WS}_{2}$, and $\mathrm{WTe}_{2}$ systems the variation of the field leaves almost unaltered both the $\mathcal{P}_{t_{2 g}, e_{g}}$ and $\mathcal{P}_{d_{x^{2}-y^{2}}, d_{z^{2}}}$ polarizations. This analysis then suggests that the applied field induces an accumulation of charge along the $c$ direction in the $\mathrm{MoSe}_{2}, \mathrm{MoTe}_{2}$, and $\mathrm{WSe}_{2}$ systems, which accounts for a significant variation of the $c$ lattice constant (Fig. 2) and the consequent instability

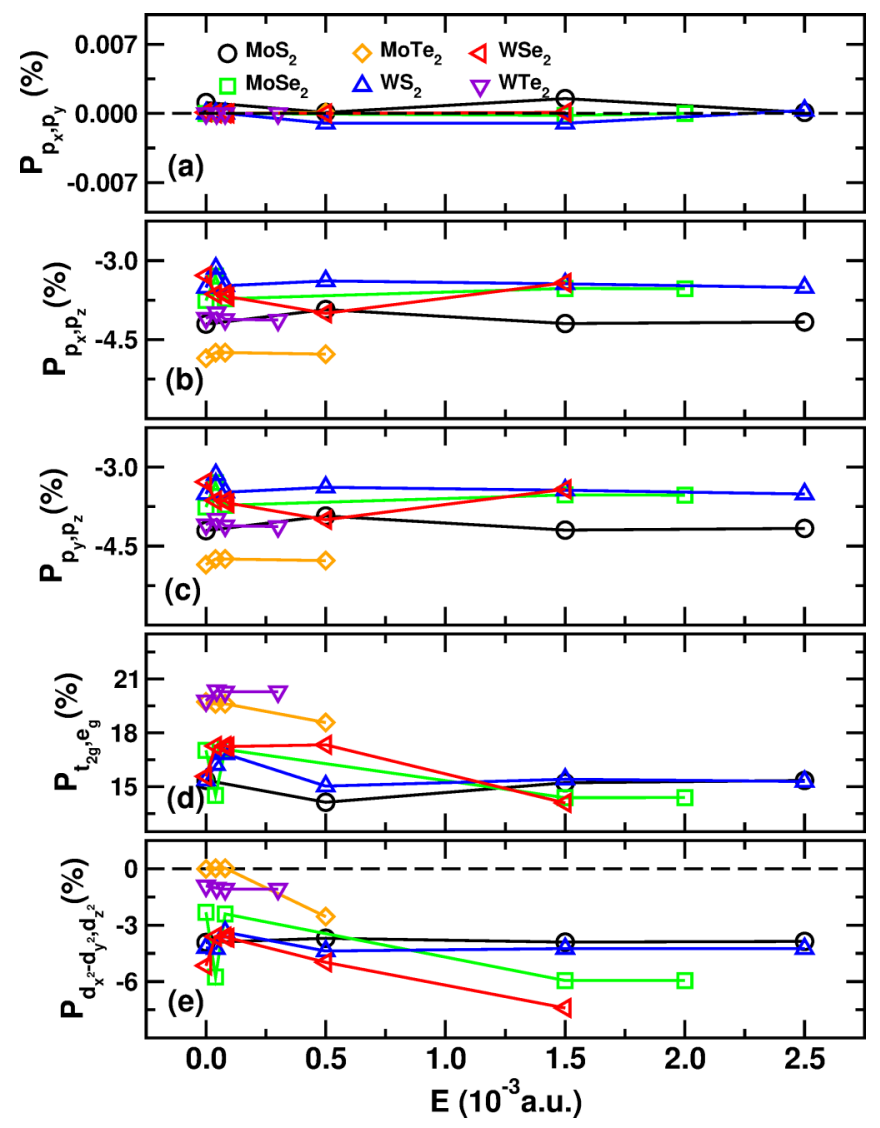

FIG. 4. Orbital polarization of the $\mathrm{MX}_{2}$ systems as a function of the electric field. An increase of the field magnitude induces a charge transfer along the direction orthogonal to the lattice planes. Legend is common to all the subplots; lines are a guide for the eye.

of the sliding modes (Fig. 3). For a convenient visualization of this effect, we report the projections of selected charge density differences in the Supplemental Material [39]. The charge redistribution may affect the $\mathrm{M}-\mathrm{X}$ bond covalency, as it has already been observed in TMD-based systems [54]. In order to quantify this effect, we calculate the bond covalency $C_{\mathrm{M}, \mathrm{X}}$ in terms of atomic participation to the electronic density of states [66]; we then consider the relation among $C_{\mathrm{M}, \mathrm{X}}$ and the polarizations involving the $d$ orbitals (Fig. 5). In $\mathrm{MoS}_{2}$, $\mathrm{MoSe}_{2}$, and $\mathrm{MoTe}_{2}$ systems, the bond covalency is nearly constant despite the large variation of the orbital polarizations; on the contrary, the covalent character of the bond is more sensitive to the charge rearrangement in $\mathrm{WS}_{2}$ and $\mathrm{WSe}_{2}$ systems. In the case of the $\mathrm{WTe}_{2}$ compound, the variation of the orbital polarization is too small to induce significant changes in the bond covalency. Correspondingly, no clear trend is observed for $\omega$ as a function of $C_{\mathrm{M}, \mathrm{X}}$ (not shown). This shows that the relation among the atomic motions (i.e., mode eigendisplacements and their frequency), the covalency, the orbital polarizations, and the atomic types forming the structure is not trivial. Indeed, the atomic types and the electronic environment in which they are embedded rule the interatomic forces which, in turn, dictate the dynamic response. In fact, the interatomic force constant tensor enters in the definition of the dynamical matrix and determines the values of the 

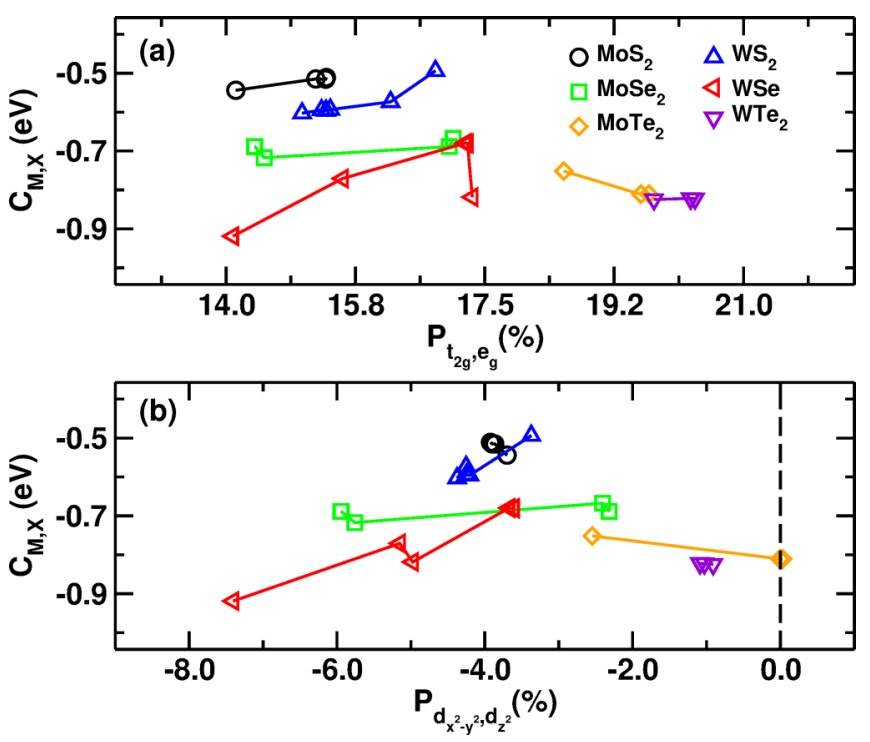

FIG. 5. M-X bond covalency as a function of the (a) $\mathcal{P}_{t_{2 g}, e_{g}}$ and (b) $\mathcal{P}_{d_{x^{2}-y^{2}}, d_{z^{2}}}$ orbital polarizations. Depending on the system, the charge rearrangement does not affect the covalent character of the bond. Legend is common to both subplots; lines are a guide for the eye.

mode frequencies of the system $[67,68]$. This leads us to consider the cophonicity [51] metric as a general descriptor able to parametrize the effect of the atomic type on such entangled electrodynamical coupling, as already observed for other physical phenomena [69-73]. The cophonicity metric $\mathrm{C}_{p h}(\mathrm{M}-\mathrm{X})$ is a measure of the relative atomic contributions to a specific range of phonon eigenfrequencies, whereas the contribution of a single atom is quantified by the atom-projected phonon density of states. To calculate $\mathrm{C}_{p h}(\mathrm{M}-\mathrm{X})$ for each compound, we select the frequency range corresponding to the sliding and breathing modes [39]. According to the definition, positive $\mathrm{C}_{p h}(\mathrm{M}-\mathrm{X})$ values indicate that the $\mathrm{M}$ and $\mathrm{X}$ ions contribute more to higher- and lower-frequency displacements, respectively; in this case, $\mathrm{M}$ cations displace faster than $\mathrm{X}$ anions when forming the global layer sliding motion. The opposite holds for negative values. A cophonicity value close to zero (perfect cophonicity) corresponds to atomic displacements in which both $\mathrm{M}$ and $\mathrm{X}$ atoms move on average at the same velocity. We observe that the cophonicity is nearly constant for low values of the electric field (Fig. 6); in this respect, it can be regarded as an intrinsic characteristic of the stable system. For each system, there is a critical electric field beyond which the cophonicity deviates significatively from the value at zero field. Correspondingly, the structure becomes highly unstable against the sliding and breathing distortions, that is, the relative layer motion is promoted in a more significant amount than what is observed for the cases at low field. Interestingly, in general, the sliding and breathing displacements are stable when perfect cophoniciy is realized $\left(\mathrm{C}_{p h}(\mathrm{M}-\mathrm{X}) \approx 0\right)$, while they become prevalently unstable when $\mathrm{C}_{p h}(\mathrm{M}-\mathrm{X})$ significantly deviates from zero; how far from zero it must be to have unstable modes depends on the system. This means that if the overall layer displacement is formed by atomic motions in which the $\mathrm{M}$ and $\mathrm{X}$ atoms move on
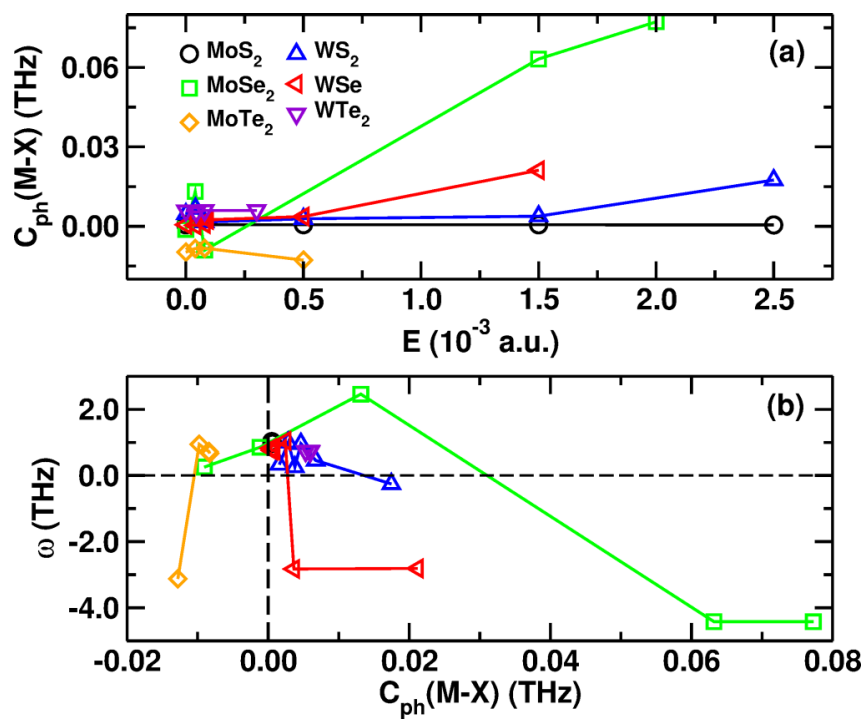

FIG. 6. (a) Cophonicity of the M-X pair as a function of the electric field. (b) Average frequency as a function of the cophonicity. Legend is common to both subplots; lines are a guide for the eye.

average at the same velocity, the sliding is favored at low electric field values; on the contrary, larger field values require that cations and anions move at a very different velocity in order to produce easy gliding of the layers. In passing, we notice that the cophonicity values here reported are similar to those realized in finite $n$-layered TMDs, where the orbital polarization and the consequent interlayer charge distribution play a relevant role in the determination of the sliding-related frequencies [50].

The presented case study can be extended by considering geometric configurations corresponding to local energy minima other than those considered in the present work; in this way, it is possible to obtain a more complete overview of the set of electric field values suitable for the selected atomic types. Moreover, atomic species and geometries other than those pertaining to the here examined TMD prototypes can be the subject of future studies. To this aim, it is possible to use the cophonicity to parametrize the nanoscale frictional response to external electric fields in large databases of chemical moieties and atomic topologies. Such parametrization can be used in machine learning engines [9] for automated research of proper combination of atomic species and geometries, to ultimately design novel tribological materials with targeted frictional response under electric field stimuli. Finally, the observed relation between the charge flow and the electric field may guide the probe and control of metal-to-insulator transition in nanomechanical motions $[74,75]$, since the transition is generally determined by subtle anisotropic rearrangements of the electronic density $[65,76,77]$.

\section{CONCLUSIONS}

We studied the effect of an electrostatic field on the nanoscale frictional properties of lamellar materials, by considering prototypical layered transition metal dichalcogenides as a case study. By applying an electric field along an axis orthogonal to the atom layers, we induce a transfer of charge 
along the same axis; this transfer does not affect significantly the covalent character of the bond, while it modifies the interatomic forces. Such modification determines the softening of the sliding modes until they become unstable above a certain critical field specific of the system. This effect is quantified by the cophonicity metric: The larger the module of the cophonicity, the more different the cation and anion velocity when forming the overall sliding motion, leading to strongly unstable phonon modes and lower friction. The present outcomes are therefore a theoretical tool for future investigations aimed to parametrize the triboresponse to electric fields against the atomic types forming the compound. The reported results also represent a starting point for further studies where different directions of the field other than that orthogonal to atomic layers are considered, in order to elucidate the effect of the field orientation on the intrinsic friction. Finally, the present study can help to predict the preferential electronic redistribution in nanomechanical devices, where local metalto-insulator transitions may occur in working conditions.

\section{ACKNOWLEDGMENTS}

This project has received funding from the European Union's Horizon2020 research and innovation programme under Grant agreement No. 721642: SOLUTION. This work has been done with the support of the Czech Science Foundation (Project No. 17-24164Y), and by the project "Novel nanostructures for engineering applications" No. CZ.02.1.01/0.0/0.0/16_026/0008396. This work was supported by The Ministry of Education, Youth and Sports from the Large Infrastructures for Research, Experimental Development and Innovations project "e-Infrastructure CZLM2018140.”
[1] M. Chhowalla, H. Shin, G. Eda, L. Li, K. Loh, and H. Zhang, Nat. Chem. 5, 263 (2013).

[2] W. Choi, N. Choudhary, G. H. Han, J. Park, D. Akinwande, and Y. H. Lee, Materials Today 20, 116 (2017).

[3] H. Schmidt, F. Giustiniano, and G. Eda, Chem. Soc. Rev. 44, 7715 (2015).

[4] W. Guo, J. Yin, H. Qiu, Y. Guo, H. Wu, and M. Xue, Friction 2, 209 (2014).

[5] J. Cumings and A. Zettl, Science 289, 602 (2000).

[6] J. P. Oviedo, S. KC, N. Lu, J. Wang, K. Cho, R. M. Wallace, and M. J. Kim, ACS Nano 9, 1543 (2015).

[7] H. Li, J. Wu, Z. Yin, and H. Zhang, Acc. Chem. Res. 47, 1067 (2014).

[8] D. Young, C. Zorman, and M. Mehregany, "Mems/nems devices and applications," in Springer Handbook of Nanotechnology, edited by B. Bhushan (Springer Berlin Heidelberg, Berlin, Heidelberg, 2007), pp. 415-442.

[9] F. Belviso, V. E. P. Claerbout, A. Comas-Vives, N. S. Dalal, F.-R. Fan, A. Filippetti, V. Fiorentini, L. Foppa, C. Franchini, B. Geisler, L. M. Ghiringhelli, A. Groß, S. Hu, J. Íñiguez, S. K. Kauwe, J. L. Musfeldt, P. Nicolini, R. Pentcheva, T. Polcar, W. Ren, F. Ricci, F. Ricci, H. S. Sen, J. M. Skelton, T. D. Sparks, A. Stroppa, A. Urru, M. Vandichel, P. Vavassori, H. Wu, K. Yang, H. J. Zhao, D. Puggioni, R. Cortese, and A. Cammarata, Inorg. Chem. 58, 14939 (2019).

[10] E. Gnecco and E. Meyer, Fundamentals of Friction and Wear on the Nanoscale, 2nd ed., NanoScience and Technology (Springer International Publishing, 2015), p. 704.

[11] E. Serpini, A. Rota, S. Valeri, E. Ukraintsev, B. Rezek, T. Polcar, and P. Nicolini, Tribol. Int. 136, 67 (2019).

[12] G. Levita, A. Cavaleiro, E. Molinari, T. Polcar, and M. Righi, J. Phys. Chem. C 118, 13809 (2014).

[13] Y. Mo, K. Turner, and I. Szlufarska, Nature (London) 457, 1116 (2009).

[14] J.-U. Lee, S. Woo, J. Park, H. C. Park, Y.-W. Son, and H. Cheong, Nat. Commun. 8, 1370 (2017).

[15] H. Bao, Y. Huang, Z. Yang, Y. Miao, P. K. Chu, K. Xu, and F. Ma, Appl. Surf. Sci. 404, 180 (2017).

[16] B. Mortazavi, A. Ostadhossein, T. Rabczuk, and A. C. T. van Duin, Phys. Chem. Chem. Phys. 18, 23695 (2016).
[17] A. Kumar, T. Staedler, and X. Jiang, Beilstein J. Nanotechnol. 4, 66 (2013).

[18] H. Wang, K. C.-C. Huang, and T. Polcar, Sci. Rep. 9, 334 (2019).

[19] A. Cammarata and T. Polcar, Nanoscale 9, 11488 (2017).

[20] F. He, X. Yang, Z. Bian, G. Xie, D. Guo, and J. Luo, Small 15, 1904613 (2019).

[21] Q. Liu, L. Li, Y. Li, Z. Gao, Z. Chen, and J. Lu, J. Phys. Chem. C 116, 21556 (2012)

[22] R. Rani, N. Jena, A. Kundu, A. De Sarkar, and K. S. Hazra, J. Appl. Phys. 127, 145101 (2020).

[23] C. Wang, W. Chen, Y. Zhang, and Q. Sun, Tribol. Lett. 59, 48 (2015).

[24] J. Wang, J. Li, C. Li, X. Cai, W. Zhu, and Y. Jia, Tribol. Lett. 61, 1 (2016).

[25] Y. J. Yun, C. S. Ah, S. Kim, W. S. Yun, B. C. Park, and D. H. Ha, Nanotechnology 18, 505304 (2007)

[26] X. Gonze, B. Amadon, G. Antonius, F. Arnardi, L. Baguet, J.-M. Beuken, J. Bieder, F. Bottin, J. Bouchet, E. Bousquet, N. Brouwer, F. Bruneval, G. Brunin, T. Cavignac, J.-B. Charraud, W. Chen, M. Côté, S. Cottenier, J. Denier, G. Geneste, P. Ghosez, M. Giantomassi, Y. Gillet, O. Gingras, D. R. Hamann, G. Hautier, X. He, N. Helbig, N. Holzwarth, Y. Jia, F. Jollet, W. Lafargue-Dit-Hauret, K. Lejaeghere, M. A. L. Marques, A. Martin, C. Martins, H. P. C. Miranda, F. Naccarato, K. Persson, G. Petretto, V. Planes, Y. Pouillon, S. Prokhorenko, F. Ricci, G.-M. Rignanese, A. H. Romero, M. M. Schmitt, M. Torrent, M. J. van Setten, B. V. Troeye, M. J. Verstraete, G. Zérah, and J. W. Zwanziger, Comput. Phys. Commun. 248, 107042 (2020).

[27] A. H. Romero, D. C. Allan, B. Amadon, G. Antonius, T. Applencourt, L. Baguet, J. Bieder, F. Bottin, J. Bouchet, E. Bousquet, F. Bruneval, G. Brunin, D. Caliste, M. Côté, J. Denier, C. Dreyer, P. Ghosez, M. Giantomassi, Y. Gillet, O. Gingras, D. R. Hamann, G. Hautier, F. Jollet, G. Jomard, A. Martin, H. P. C. Miranda, F. Naccarato, G. Petretto, N. A. Pike, V. Planes, S. Prokhorenko, T. Rangel, F. Ricci, G.-M. Rignanese, M. Royo, M. Stengel, M. Torrent, M. J. van Setten, B. V. Troeye, M. J. Verstraete, J. Wiktor, J. W. Zwanziger, and X. Gonze, J. Chem. Phys. 152, 124102 (2020). 
[28] M. Torrent, F. Jollet, F. Bottin, G. Zérah, and X. Gonze, Comput. Mater. Sci. 42, 337 (2008).

[29] D. R. Hamann, Phys. Rev. B 88, 085117 (2013).

[30] X. Gonze, B. Amadon, P.-M. Anglade, J.-M. Beuken, F. Bottin, P. Boulanger, F. Bruneval, D. Caliste, R. Caracas, M. Côté, T. Deutsch, L. Genovese, P. Ghosez, M. Giantomassi, S. Goedecker, D. Hamann, P. Hermet, F. Jollet, G. Jomard, S. Leroux, M. Mancini, S. Mazevet, M. Oliveira, G. Onida, Y. Pouillon, T. Rangel, G.-M. Rignanese, D. Sangalli, R. Shaltaf, M. Torrent, M. Verstraete, G. Zerah, and J. Zwanziger, Comput. Phys. Commun. 180, 2582 (2009).

[31] X. Gonze, G.-M. Rignanese, M. Verstraete, J.-M. Beuken, Y. Pouillon, R. Caracas, F. Jollet, M. Torrent, G. Zerah, M. Mikami, P. Ghosez, M. Veithen, J.-Y. Raty, V. Olevano, F. Bruneval, L. Reining, R. Godby, G. Onida, and D. H. D.C. Allan, Z. Kristallogr. - Cryst. Mater. 220, 558 (2005).

[32] X. Gonze, Z. Kristallogr. - Cryst. Mater. 220, 558 (2005).

[33] B. Schönfeld, J. J. Huang, and S. C. Moss, Acta Crystallogr. B 39, 404 (1983).

[34] V. Kalikhman, Inorg. Mater. 19, 957 (1983).

[35] L. Brixner, J. Inorg. Nucl. Chem. 24, 257 (1962).

[36] W. Schutte, J. D. Boer, and F. Jellinek, J. Solid State Chem. 70, 207 (1987).

[37] V. L. Kalikhman, Neorganicheskie Materialy 19, 1060 (1983).

[38] A. A. Yanaki and V. A. Obolonchik, Inorg. Mater. 9, 1855 (1973).

[39] See Supplemental Material at http://link.aps.org/supplemental/ 10.1103/PhysRevB.102.155433, section "Benchmark of van der Waals corrections" which includes Refs. [78-82], section " $\Gamma-A$ Phonon Band Dispersion in the $\mathrm{MX}_{2}$ model systems," section "Sliding and breathing mode frequencies as a function of the applied field," section "Charge density difference projections, and section "Integration intervals for the calculation of the cophonicity."

[40] J. P. Perdew, K. Burke, and M. Ernzerhof, Phys. Rev. Lett 77, 3865 (1996).

[41] A. D. Becke and E. R. Johnson, J. Chem. Phys. 124, 221101 (2006).

[42] R. W. Nunes and X. Gonze, Phys. Rev. B 63, 155107 (2001).

[43] R. W. Nunes and D. Vanderbilt, Phys. Rev. Lett. 73, 712 (1994).

[44] M. Mehregany, S. Senturia, J. Lang, and P. Nagarkar, IEEE Trans. Electron Devices 39, 2060 (1992).

[45] G. Meng, Y. Cheng, L. Chen, Y. Chen, and K. Wu, in 2013 IEEE International Conference on Solid Dielectrics ICSD 2013: Bologna, Italy, 30 June - 4 July 2013; Vol. 1, IEEE International Conference on Solid Dielectrics; 11 (IEEE, Piscataway, NJ, 2013), pp. 662-665.

[46] J. Guo, J. Gallegos, A. Tom, and D. Fan, ACS Nano 12, 1179 (2018).

[47] Y. Yoshizumi, T. Honegger, K. Berton, H. Suzuki, and D. Peyrade, Small 11, 5630 (2015).

[48] L. Zhang, Z. Xiao, X. Chen, J. Chen, and W. Wang, ACS Nano 13, 8842 (2019).

[49] A. Togo and I. Tanaka, Scr. Mater. 108, 1 (2015).

[50] A. Cammarata and T. Polcar, Phys. Chem. Chem. Phys. 18, 4807 (2016).

[51] A. Cammarata and T. Polcar, Inorg. Chem. 54, 5739 (2015).

[52] A. Cammarata and T. Polcar, Phys. Rev. B 96, 085406 (2017).
[53] A. Cammarata, P. Nicolini, K. Simonovic, E. Ukraintsev, and T. Polcar, Phys. Rev. B 99, 094309 (2019).

[54] A. Cammarata and T. Polcar, Phys. Rev. B 102, 085409 (2020).

[55] When the eigendisplacement of a phonon mode is unstable, the corresponding eigenfrequency is an imaginary number; however, it is instead usually indicated by a negative real number for convenience of representation.

[56] S. Zhou, J. Han, S. Dai, J. Sun, and D. J. Srolovitz, Phys. Rev. B 92, 155438 (2015).

[57] T. Liang, W. G. Sawyer, S. S. Perry, S. B. Sinnott, and S. R. Phillpot, Phys. Rev. B 77, 104105 (2008).

[58] G. Levita, E. Molinari, T. Polcar, and M. C. Righi, Phys. Rev. B 92, 085434 (2015).

[59] A. V. Lebedev, I. V. Lebedeva, A. M. Popov, A. A. Knizhnik, N. A. Poklonski, and S. A. Vyrko, Phys. Rev. B 102, 045418 (2020).

[60] B. J. Irving, P. Nicolini, and T. Polcar, Nanoscale 9, 5597 (2017).

[61] W. Jo, I. Eom, E. C. Landahl, S. Lee, and C.-J. Yu, Rev. Sci. Instrum. 87, 035107 (2016).

[62] N. Casaretto, D. Schaniel, P. Alle, E. Wenger, P. Parois, B. Fournier, E.-E. Bendeif, C. Palin, and S. Pillet, Crystallogr. Sect. 73, 696 (2017).

[63] T. Ors, N. Ranc, M. Pelerin, V. Michel, V. Favier, O. Castelnau, C. Mocuta, and D. Thiaudière, J. Synchrotron Radiat. 26, 1660 (2019).

[64] A. Cammarata, RSC Adv. 9, 37491 (2019).

[65] A. Cammarata and J. M. Rondinelli, Phys. Rev. B 87, 155135 (2013).

[66] A. Cammarata and J. M. Rondinelli, J. Chem. Phys. 141, 114704 (2014).

[67] D. M. Wallace, Thermodynamics of Crystals (John Wiley \& Sons Inc, 1972).

[68] J. M. Ziman, Electrons and Phonons: The Theory of Transport Phenomena in Solids (Oxford University Press, 2001).

[69] T. Hu, M. Hu, Z. Li, H. Zhang, C. Zhang, J. Wang, and X. Wang, J. Phys. Chem. A 119, 12977 (2015).

[70] Z. Lu, C. Chen, Z. M. Baiyee, X. Chen, C. Niu, and F. Ciucci, Phys. Chem. Chem. Phys. 17, 32547 (2015).

[71] R. Fei, W. Kang, and L. Yang, Phys. Rev. Lett. 117, 097601 (2016).

[72] Z. Hu, Y. Ding, X. Hu, W. Zhou, X. Yu, and S. Zhang, Nanotechnology 30, 252001 (2019).

[73] Y. Fan, X. Liu, J. Wang, H. Ai, and M. Zhao, Phys. Chem. Chem. Phys. 20, 11369 (2018).

[74] J. Wei, Z. Wang, W. Chen, and D. H. Cobden, Nat. Nanotechnol. 4, 420 (2009).

[75] D. Singh and B. Viswanath, J. Mater. Sci. 52, 5589 (2017).

[76] S. Lv, H. Li, Z. Wang, L. Han, Y. Liu, X. Liu, and J. Meng, Appl. Phys. Lett. 99, 202110 (2011).

[77] A. I. Poteryaev, M. Ferrero, A. Georges, and O. Parcollet, Phys. Rev. B 78, 045115 (2008).

[78] S. Grimme, J. Comput. Chem. 25, 1463 (2004).

[79] I. V. Lebedeva, A. V. Lebedev, A. M. Popov, and A. A. Knizhnik, Comput. Mater. Sci. 128, 45 (2017).

[80] S. Grimme, J. Comput. Chem. 27, 1787 (2006).

[81] A. Ambrosetti and P. L. Silvestrelli, Phys. Rev. B 85, 073101 (2012).

[82] P. L. Silvestrelli, J. Chem. Phys. 139, 054106 (2013). 